\title{
A Case Report on Autoimmune Polyendocrine SyndromeType I
}

\author{
MD. YUSUF ALI, ${ }^{1}$ HM RASHIDUZZAMAN, ${ }^{2}$ MAHADI MASUD, ${ }^{3}$ MD. ABDUL WADUD, ${ }^{4}$ SADIA QUADIR, ${ }^{2}$ SK. ABDUL \\ FATTAH, ${ }^{5}$ MD. ENAMUL KARIM ${ }^{6}$
}

\begin{abstract}
Autoimmune polyendocrine syndrome type I (APS-I) is a rare autosomal recessive disorder characterized by autoimmune multiorgan dysfunction. The major components of APS type I are chronic mucocutaneous candidiasis,hypoparathyroidism and Addison's disease.To establish this syndrome, at least two of these conditions have to be present. We report here one of such case, a 15-year old boy who presented with features of chronic mucocutaneous candidiasis, hypoparathyroidism, primary hypothyroidism,nail dystrophy and dental enamel hypoplasia that were consistent with APS-I.
\end{abstract}

Keywords:Autoimmune polyendocrine syndrome type I, chronicmucocutaneous candidiasis, hypoparathyroidism.

\section{Introduction}

Autoimmune polyendocrine syndrome type 1 (APS-1), which is also termed autoimmune polyendocrinopathy-candidiasisectodermal dystrophy (APECED), is a rare disorder and is inherited in an autosomal recessive fashion, caused by mutations in the autoimmune regulator gene (AIRE) and characterized by the failure of several endocrine glands as well as nonendocrine organs. ${ }^{1}$ APS type 1 appears to occur worldwide. Approximately 500 patients have been reported so far. APS type 1 has been noted to occur more frequently among homogeneous populations, such as those in Finland, northern Italy, Norway and Sardinia, as well as among Iranian Jews. ${ }^{2-4}$ The major components of APS type 1 are chronic mucocutaneous candidiasis, chronic hypoparathyroidism, and Addison's disease. These are collectively called Whitaker's triad, which is pathognomonic for APS type $1 .{ }^{5}$ The spectrum of associated minor clinical diseases include primary hypothyroidism, type 1 diabetes, nail dystrophy and dental enamel hypoplasia. In general, the first manifestation usually occurs in the childhood and the complete evolution of the three main diseases takes place in the first 20 year of

1. Professor \& Head,Dept. of Medicine, Faridpur Medical College Hospital, Faridpur.

2. Registrar, Dept. of Medicine, Faridpur Medical College Hospital, Faridpur.

3. Medical officer,Dept. of Medicine, Faridpur Medical College Hospital, Faridpur.

4. Assistant Registrar,Dept. of Medicine, Faridpur Medical College Hospital, Faridpur.

5. Associate Professor, Dept. of Medicine, Faridpur Medical College Hospital, Faridpur.

6. Professor,Dept. of Medicine, Dhaka Medical College Hospital, Dhaka.

Correspondence: Professor Dr. Md. Yusuf Ali, Professor \&Head, Dept. of Medicine, Faridpur Medical College Hospital, Faridpur. E-mail: yusufali1958@yahoo.com. age, whereas other accompanying diseases continue to appear until at least the fifth decade. In a majority of cases, candidiasis is the first clinical manifestation to appear, usually before the age of 5 year, followed by hypoparathyroidism, usually before the age of 10 year and later by Addison's disease that occur usually before 15 year of age. Overall, the three main components of APS type 1 occur in a fairly precise chronological order, but they are present together in only about one third to one half of the cases. It has been reported that the earlier the first component appears, the more likely it is that multiple components will develop; conversely, patients who have late manifestations of the disease are likely to have fewer components. ${ }^{6,7}$ Therefore, patients with APS-1 should be followed-up on a regular basis because the majority of the above conditions develop later in the course of the disease. ${ }^{8}$ Classical diagnosis is made by presence of atleast 2 major components or only one component if a sibling has already been diagnosed. Diagnostic criterion of having at least two elements of this triad would leave many cases missed. In some cases the minor components dominate with none of the triad present. If APS type 1 is suspected, genetic analysis of the AIRE gene may be helpful to confirm diagnosis, especially in atypical clinical presentations. Autoantibodies especially IFNá- and IFN-ù are the diagnostic tool for APS type 1, especially in cases where mutational analysis is complicated (for example, large deletions, duplications, or mutations in regulatory or intronic regions). ${ }^{9}$

\section{Case Report}

A 15 year old boy, hailing from Boalmari, Faridpur, was a known case of hypothyroidism for last 11 years with irregular intake of thyroxine.He was nondiabetic, normotensive and got admitted on $3^{\text {rd }}$ April, 2013in FMCH with repeated convulsion forlast one month. Each of the attack started from his right hand andthen became generalized and comprised a tonic and clonic phase. There 
were 5-6 episodes of convulsion per day and each episode persisted for 5-10 minutes followed by loss of consciousness for about 30 minutes to 1 hour. Convulsion was associated with tongue bite and urinary incontinence and not associated with fever, headache and vomiting.He had no history of neck surgery, head injury orsyncopal attack. He developed recurrent skin infection in different parts of his body since childhood.He had a cataract surgery of his right eye five months back and developed blurring of vision in his left eye for few months. On examination he was ill looking with stunted growth and his body built was below average, mildly anaemic, pulse-76 beats/minute, blood pressure-90/60 $\mathrm{mm}$ of $\mathrm{Hg}$. There was no postural drop of blood pressure. Trousseau's sign was positive.His thyroid gland was not enlarged.Fundoscopy reveals normal
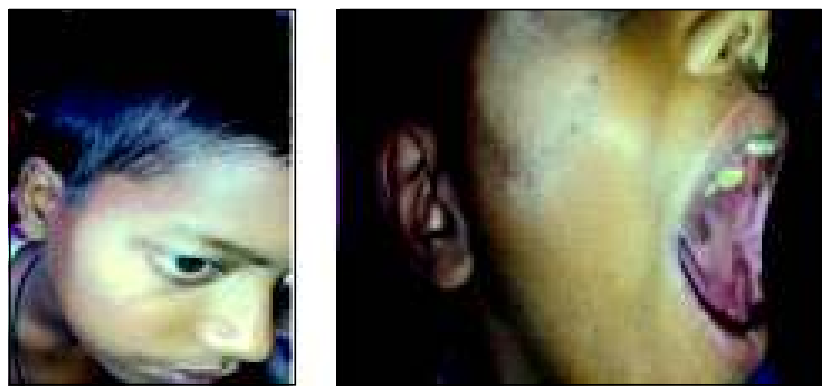

Fig.-1: Chronic mucocutaneous candidiasis

in right side and left side of fundus could not bevisible due to early stage of cataract. Fungal infectionwas present in oral cavity and different parts of body like cheek and trunk. Nail dystrophy and dental enamel hypoplasia were present in almost all of the nails and teeth respectively.His bowel and bladder habit was normal.

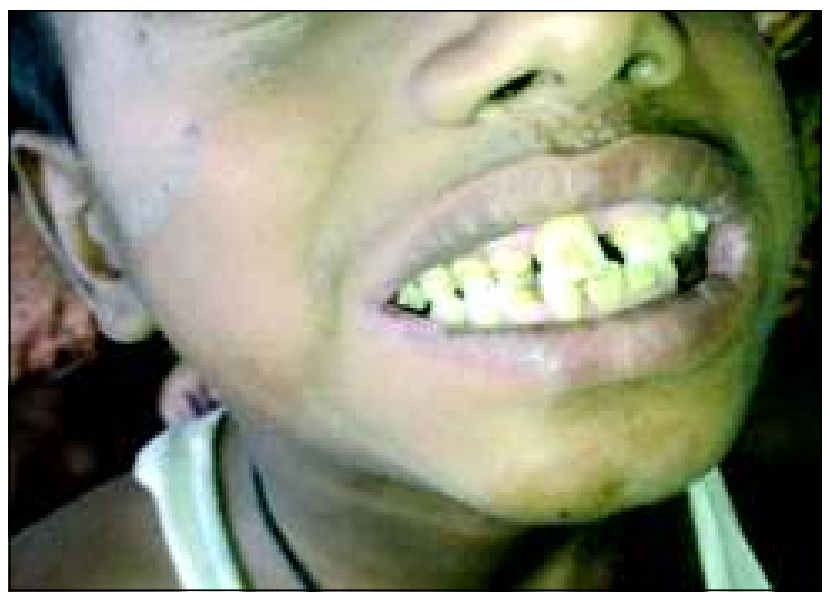

Fig.-2: Dental enamel hypoplasia

His serum TSH level $(75 \mu \mathrm{IU} / \mathrm{ml})$ was high, serum PTH (< $1 \mathrm{pg} / \mathrm{ml})$ and calcium $(4.6 \mathrm{mg} / \mathrm{dl})$ were low, serum albumin

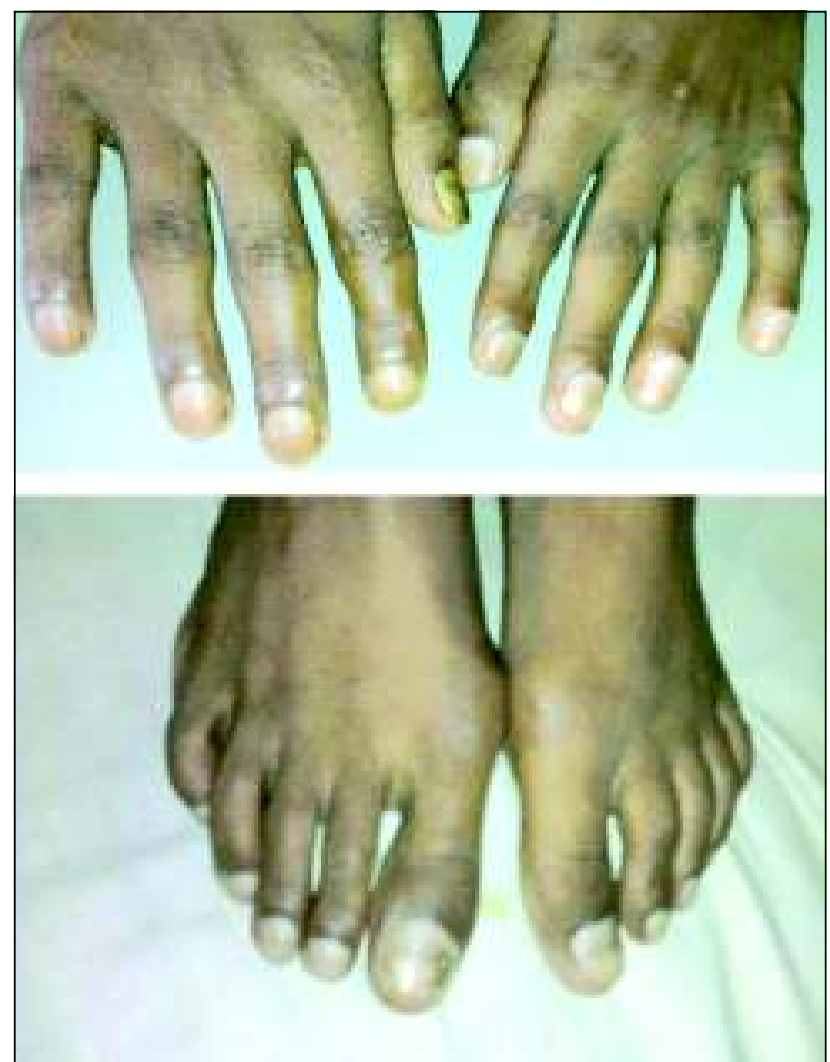

Fig 3: Nail dystrophy

(40.34 gm/dl) and random blood sugar $(92 \mathrm{mg} / \mathrm{dl})$ were normal. CT scan of brain showed diffuse nearly symmetrical calcification of both dentate nuclei, both basal ganglia region, both thalami, both coronaradiate, sub-cortical region of both frontal, parietal, temporal and occipital lobes. His complete blood count, serum creatinine, serum electrolytes, U.S.G of whole abdomen, E.C.G and chest X-ray P/A view findings were normal.

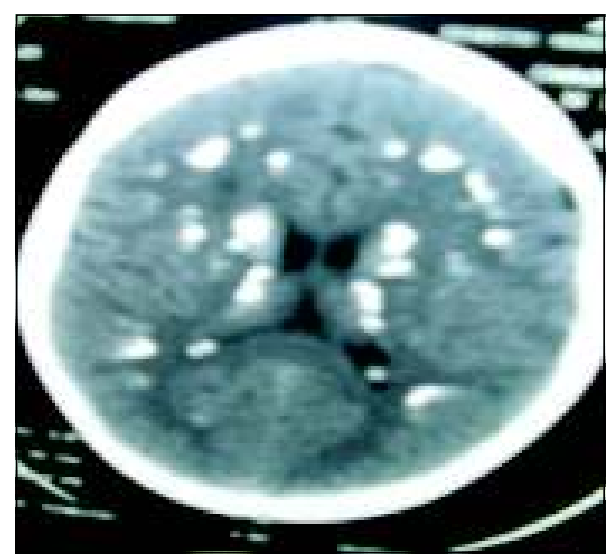

Fig.-4: CT scan of brain showed diffuse nearly symmetrical calcification of both dentate nuclei, both basal ganglia region, both thalami, both corona radiate, sub-cortical region of both frontal, parietal, temporal and occipital lobes. 


\section{Discussion}

The reported patient presented with hypothyroidism for last 11 years, chronic mucocutaneous candidiasis since childhood and features of hypoparathyroidism (convulsion for last one month, positive Trousseau's sign and calcification in CT scan of brain). He had also nail dystrophy and dental enamel hypoplasia. Chronic mucocutaneous candidiasis (CMC) is themost common manifestation in APS1. It is present in almost $100 \%$ of the patients. In most cases, $\mathrm{CMC}$ is the first of the major components of APS-1 to appear, often occurring before age 5 , and its severity is variable. It preferably affects the oral mucosa causing a mild form of intermittent angular cheilitis. More severe cases include inflammation of most of the oral mucosa, hyperplastic CMC with thick white or grey plaques of yeast and hyperkeratosis, and atrophic form with thin mucosa and leukoplakic areas. ${ }^{10,}$

11 Our patient presented withchronic mucocutaneous candidiasis in oral cavity and different parts of body like cheek and trunk since childhood. CMC was present in a case report made by Kibirige and Kambugu. ${ }^{12}$

Another cardinal component,hypoparathyroidism,is usually the first endocrine disorder to develop during the course of APS 1 and it has been reported in 70\%-93\% of the cases. ${ }^{13}$ It varies according to gender, affecting $98 \%$ of female patients, but only $71 \%$ of male patients. Interestingly, when adrenal insufficiency is thefirst endocrinopathy, susceptibility tohypoparathyroidism appears to bereduced. ${ }^{14}$ Our patient had hypoparathyroidism as he presented with repeated attack of convulsion and positive Trousseau's sign. We also found very low parathyroid hormone and calcium level in blood and CT scan showed diffuse calcification in brain.Hypoparathyroidism was also present in case report made by Kibirige and Kambugu. ${ }^{12}$

Our patient was a known case hypothyroidism for last 11 years. In APS type 1 hypothyroidism is relatively uncommon, affecting no more than $30 \%$ of the APS- 1 patients. It develops more often following puberty and by middle age, usually before the age of $30^{10}$. Hypothyroidism was present in case report made by Ming-Chen et al. ${ }^{15}$

Among the minor clinical disease the reported patient also presented with nail dystrophy and dental enamel hypoplasia. Primary adrenal insufficiency or Addison'sdisease ispresent approximately in 60\%-100\% cases, with peakincidence at around 12 years of age. It is a life threatening condition that should be rapidly recognizedand treated. Symptoms are fatigue, weight loss, salt craving, hypotension, abdominal pain and increased pigmentation of the skin. ${ }^{13}$ Our patient had no feature of Addison'sdisease. Addison's disease was also absent in case report made by Kibirige and Kambugu. ${ }^{12}$
The presentation of our case was typical.He had two major components of classical triad and diagnosed as a case of APS type 1. Autoimmune polyendocrine syndrome type 1 (APS-1), is an extremely rare and frequently debilitating disorder of childhood. It is an autosomal recessive condition and a monogenic disorder linked to a defect of the AIRE gene located on chromosome $21 \mathrm{q} 22.3 .{ }^{16} \mathrm{~A}$ clinical diagnosis ofAPS-1 classically requires the presence of two of the three cardinal components: chronic mucocutaneous candidiasis, hypoparathyroidism and Addison's disease ${ }^{6}$. Early immunogenetic testing for the different mutations of the AIRE gene and human leukocyte antigen (HLA) typing are essential for identification of patients at risk. This is because APS-1 occurs sporadically or in siblings ${ }^{11}$. HLA-A28 has been demonstrated to occur more frequently in patients with APS-1 than in normal controls. Generally, HLA-A3 is mostly observed in patients with APS- $1 .{ }^{17}$ However, these tests are not readily available in most resource-limited settings.

\section{Conclusion}

Early recognition of multi- organ autoimmunedisease is the best way to minimize associatedmorbidity and mortality. The aim of this case report is to grow awareness among health related personale about APS-1.A thorough history and physicalexamination should always be performed and a highindex of suspicion should be maintained.

\section{Conflict of Interest : None}

\section{References}

1. Strachan MWJ, Walker BR. Endocrine disease. In: Colledge NR, Walker BR, Ralston SH eds. Davidson's Principles \& Practice of Medicine. $21^{\text {st }}$ ed. India: Churchill Livingstone, 2010: 794 .

2. Ahonen P, Myllarniemi S, Sipila I, Perheentupa J. Clinical variation of autoimmune polyendocrinopathy-candidiasisectodermal dystrophy (APECED) in a series of 68 patients. N Engl J Med. 1990; 322(26):1829-36.

3. Betterle C, Greggio N, Volpato M. Autoimmune polyglandular syndrome type I. JClinEndocrinolMetab. 1998; 83(4): 1049-55.

4. Myhre AG, Halonen M, Eskelin P. Autoimmune polyendocrine syndrometype 1 (APS I) in Norway. ClinEndocrinol (Oxf). 2001; 54(2):211-17.

5. Hans DO. Primary Immunodeficiency Diseases: A Molecular and Genetic $2^{\text {nd }}$ edition Ann. N.Y. Acad. Sci. 1246 (2011) 77-91.

6. Neufeld M, MacLarenNK, Blizzard RM. Two types of autoimmune Addison's disease associated with different polyglandular autoimmune (PGA) syndromes. Medicine. $1981 ; 60: 355-62$.

7. Ahonen P, Myllarniemi S, Sipila I, Perheentupa J. Clinical variation of autoimmune polyendocrinopathy-candidiasis- 
ectodermal dystrophy (APECED) in a series of 68 patients. N Engl J Med. 1990; 322:1829 -36.

8. Manuela D, George J. Polyglandular autoimmune syndromes: immunogeneticsand long-term follow-up. J Clin Endocrinol Metab. 2003; 88(7):2983-92.

9. Owen C, Cheetham T. Diagnosis and management of polyendocrinopathysyndromes. EndocrinolMetabClin N Am. 2009; 38(2):419-36.

10. Betterle C, Greggio NA, Volpato M. Clinical review 93: autoimmune polyglandular syndrome type 1. J Clin Endocrinol Metab. 1998; 83(4):1049-55.

11. Perheentupa J. Autoimmune polyendocrinopathycandidiasis-ectodermal dystrophy. J ClinEndocrinolMetab. 2006;91(8):2843-50.

12. Kibirige D, Kambugu F. Autoimmune polyglandular syndrome type 1 in a 12-year-old Ugandan girl. Jemdsa. 2013; 18(1):65-67.

13. Soderbergh A, Myhre AG, Ekwall O, Gebre-Medhin G, Hedstrand H, Landgren E, et al. Prevalence and clinical associations of 10 defined autoantibodies in autoimmune polyendocrine syndrome type I. J ClinEndocrinolMetab. 2004; 89(2): 557-62.

14. Gylling M, Kaariainen E, Vaisanen R, Kerosuo L, Solin ML, Halme L, et al. The hypoparathyroidism of autoimmune polyendocrinopathy- candidiasis-ectodermal dystrophy protective effect of male sex. J ClinEndocrinolMetab. 2003; 88(10): 4602-8.

15. Ming-Chen H, Hsin-Dean C, Du-An W. Polyglandular Autoimmune Syndromes - A Case Report. Tzu Chi Med J.2006; 18:385-388.

16. Noriko S, Gretel N, Georg A. Clearing the AIRE: on the pathophysiological basis of the autoimmune polyendocrinopathy syndrome type- 1 . Endocrinol Metab Clin N Am. 2009; 38(2): 273-88.

17. Ahonen P, Koskimies S, Lokki M, et al. The expression of autoimmune polyglandular disease type I appears associated with several HLA-A antigens but not with HLADR. J ClinEndocrinolMetab. 1988; 66(6):1152-57. 